Revue scientifique sur la conception et l'aménagement de l'espace

\title{
Étude du développement de l'agglomération lyonnaise depuis 1950
}

Le SIG, un outil au service de l'expertise territoriale

Study of the development of the Lyon area since 1950 - The GIS, a tool in the service of territorial expertise

\section{Nicolas Ferrand}

\section{OpenEdition \\ Journals}

Édition électronique

URL : http://journals.openedition.org/paysage/15624

DOI : $10.4000 /$ paysage. 15624

ISSN : 1969-6124

\section{Éditeur :}

École nationale supérieure du paysage de Versailles-Marseille, Institut national des sciences appliquées Centre Val de Loire - École de la nature et du paysage, École nationale supérieure d'architecture et de paysage de Bordeaux, École nationale supérieure d'architecture et de paysage de Lille, Agrocampus Angers

\section{Référence électronique}

Nicolas Ferrand, "Étude du développement de l'agglomération lyonnaise depuis 1950 », Projets de paysage [En ligne], 8 | 2012, mis en ligne le 13 juillet 2012, consulté le 11 mars 2021. URL : http:// journals.openedition.org/paysage/15624; DOI : https://doi.org/10.4000/paysage.15624

Ce document a été généré automatiquement le 11 mars 2021

Projets de paysage 


\title{
Étude du développement de
} l'agglomération lyonnaise depuis 1950

\author{
Le SIG, un outil au service de l'expertise territoriale \\ Study of the development of the Lyon area since 1950 - The GIS, a tool in the \\ service of territorial expertise
}

\section{Nicolas Ferrand}

1 L'urbanisation de l'agglomération lyonnaise et ses dynamiques sont connues. Certains traits forts de ce développement urbain ont été identifiés tôt, alors qu'ils n'en étaient qu'à leurs prémices. C'est le cas, par exemple, de la désindustrialisation du centre de l'agglomération (Laferrère, 1960; Bonneville, 1975). De même, les travaux liés à la planification ont permis de prendre conscience, dès la fin des années 1950, du risque d'étalement en tache d'huile de l'urbanisation. La nécessité de structurer l'agglomération, autour de pôles moteurs et d'axes structurants, et de préserver les zones boisées est pointée dans le plan d'urbanisme directeur de $1962^{1}$ (Groupement d'urbanisme de la région lyonnaise, 1962). L'analyse du corpus bibliographique traitant de cette thématique (dont la quasi-totalité des mémoires universitaires soutenus depuis 1945) a permis de constater que, si des analyses fines avaient été réalisées dans quelques cas particuliers, aucune étude n'avait encore été faite sur l'ensemble de l'agglomération sur un pas de temps conséquent.

2 Les avancées présentées ici sont de deux ordres. Le premier point concerne le développement d'une méthodologie complexe, dont la mise au point fait partie, intrinsèquement, de la problématique de départ. Celle-ci mobilise d'importantes quantités de sources hétérogènes et inédites, dont l'exploitation donne lieu à la production de données à la fois cartographiques et statistiques. En second lieu, il s'agit bien sûr de la production proprement dite d'une information en grande majorité nouvelle, sur l'urbanisation de l'agglomération lyonnaise. Les derniers développements de cet outil, ainsi que les résultats obtenus récemment, sont le fruit d'une étroite 
coopération avec l'Agence d'urbanisme pour le développement de l'agglomération lyonnaise, qui voit dans celui-ci un apport appréciable dans sa mission quotidienne d'expertise et de veille sur la question urbaine.

\section{Concevoir un outil d'analyse de l'urbanisation de l'agglomération lyonnaise}

3 Le postulat de départ de ce projet a été d'appréhender l'espace urbain comme un ensemble d'unités de conception, "formes de l'œuvre architecturale ou urbanistique que l'on peut individualiser par leurs régularités, leurs dispositions ou leur composition urbaine " (Gauthiez, 2003). Cette dimension de l'urbanisation a été retenue, car elle permet d'identifier, sur la carte topographique, le plan cadastral ou la photographie aérienne des unités urbaines en fonction de leurs caractéristiques morphologiques. Plusieurs types d'unités ont ainsi été retenus. Il s'agit, pour les plus importantes, des unités collectives, pavillonnaires, d'activités et d'équipements. Ces unités morphologiques correspondent toutes à un tènement parcellaire identifié sur le plan cadastral vectorisé. Ce tènement, objet géographique à part entière, a des caractéristiques qui lui sont propres : a minima son périmètre et sa surface. Il est également caractérisé par un contenu particulier : des bâtiments, des infrastructures, des logements, etc. Une procédure d'urbanisme, qu'il s'agit alors d'identifier, a pu y avoir été réalisée. Celle-ci permettra d'apporter des éléments de compréhension sur la réalisation d'un nouveau composant urbain.

\section{Mise en œuvre d'un $\mathrm{SIG}^{2}$ historique}

Documenter les processus de création de ces unités morphologiques a nécessité d'identifier les documents administratifs qui ont permis leur réalisation, sur l'ensemble de la période étudiée. Il a notamment été nécessaire de dépouiller environ 5500 dossiers de lotissements et 200 dossiers de ZAC. Cette démarche, puisqu'elle se base sur l'utilisation de sources parfois constituées il y a plus de soixante ans, s'appuie sur des méthodes d'investigation jusqu'alors utilisées en sciences historiques (Demangeon, 1907). Elle permet de mettre en évidence des processus particuliers sur le long terme, tant en ce qui concerne les jeux d'acteurs que l'évolution de la structure parcellaire et, in fine, de la morphologie urbaine (Panerai, 1997 ; Rouleau, 1985). Le dépouillement de ces dossiers administratifs n'est cependant qu'un préalable pour identifier les processus et manifestations de l'urbanisation de l'agglomération lyonnaise depuis 1945.

5 L'ensemble des données identifiées a été centralisé dans un SIG historique. Cet outil est particulièrement efficace pour traiter des données hétérogènes, de nature et d'échelle différentes (Arnaud, 2008). Les sources doivent souvent être remaniées, « de façon à en rendre cohérentes les informations spatiales, qu'il s'agisse des données planimétriques et cartographiques, de la matérialité architecturale, et des données issues des textes ou sources sérielles. » (Gauthiez, Zeller dans Panzeri, Farrugia [dir.], 2009). Utilisé pour prendre en compte la dimension temporelle, le SIG constitue également un outil efficace pour, d'une part, réaliser des études du territoire à une échelle fine et, d'autre part, observer le développement urbain dans le temps (Gregory, Kemp, Mostern, 2001). Il s'agit donc d'observer dans le temps l'évolution de tènements parcellaires qui, en plus d'un demi-siècle, ont parfois connu plusieurs mutations successives. 
6 Le SIG historique offre la possibilité de croiser l'ensemble des sources pour s'assurer de la véracité de l'information recueillie. En effet, une autorisation préfectorale sur un dossier de lotissement n'implique pas forcément que l'opération ait été immédiatement réalisée par la suite. Il est donc important de vérifier que la division parcellaire a été effectuée et que les constructions prévues dans le projet d'aménagement ont bien été réalisées. Cette vérification est aisée dans le SIG, en comparant immédiatement la structure parcellaire contemporaine au projet autorisé. La carte topographique permet de vérifier si le projet a été réalisé au moment de son autorisation ou si, au contraire, sa réalisation a été différée dans le temps. En outre, l'ensemble des sources tierces (cartes, photos aériennes, etc.) permet d'identifier des unités morphologiques qui n'ont pas été prises en compte dans les dossiers administratifs, et donc de combler des lacunes dans des séries souvent incomplètes. L'information attribuée à ces unités morphologiques est ainsi plus ténue, même s'il reste possible d'obtenir, a minima, leur période de réalisation.

\section{Constituer et alimenter des bases de données de référence}

7 Des bases de données ont été constituées dans le SIG historique, à partir du dépouillement systématique de séries archivées (dossiers administratifs : lotissements et ZAC). D'autres bases de données ont été mobilisées et homogénéisées, pour correspondre au standard développé dans cet outil. Il s'agit notamment de l'identification des emprises de plus de 2000 opérations de logements locatifs sociaux consignées dans l'atlas du logement locatif et social ${ }^{3}$ (Agence d'urbanisme pour le développement de l'agglomération lyonnaise, 2007). Au final, l'ensemble de ces bases de données permet d'identifier, au sein de l'agglomération lyonnaise, l'ensemble des unités morphologiques, qu'il s'agisse d'unités pavillonnaires, collectives (sociales, privées et mixtes), d'activités, militaires, d'équipements, d'infrastructures, ou même de réseaux ferroviaires et routiers.

8 La nécessité de disposer d'une source de référence incontestable pour l'ensemble de la période d'étude a conduit à rassembler la totalité de la cartographie produite par l'Institut géographique national, depuis 1900, aux 1/25 000e et 1/50 000e. Les cartes ont été capitalisées sous forme d'images, après numérisation. Elles ont systématiquement été géoréférencées dans le SIG et constituent un atlas permettant de visualiser tout ou partie de l'agglomération à chaque période ayant fait l'objet d'une mise à jour par l'IGN ${ }^{4}$.

9 L'utilisation de ces seules bases de données, en l'état, n'est pas satisfaisante. Tout d'abord, plusieurs bases de données peuvent décrire indépendamment l'état d'un même tènement parcellaire à des époques différentes. La désindustrialisation, particulièrement importante dans le centre de l'agglomération, induit de disposer d'informations distinctes, d'une part, sur la présence de zones d'activités dans les années 1950 et, d'autre part, sur la réalisation de programmes collectifs à leur place, à partir des années 1970.

10 D'autre part, les bases constituées à partir de dossiers administratifs demandent un degré de lecture supplémentaire. Un dossier de lotissement, par exemple, ne conduit pas obligatoirement à la réalisation de logements pavillonnaires, mais peut avoir pour objet la construction de logements collectifs, de locaux d'activités ou d'équipements. Réciproquement, sur la totalité des logements pavillonnaires construits dans la 
communauté urbaine de Lyon depuis 1950 , environ $70 \%$ ont été construits dans le cadre de lotissements, $8 \%$ dans le cadre de ZAC et $22 \%$ en diffus, dans le cadre du seul permis de construire. En outre, plusieurs dossiers administratifs successifs peuvent avoir été déposés et approuvés, alors qu'il s'agit d'une seule réalisation. C'est le cas, par exemple, d'une des zones industrielles de Meyzieu, conçue au départ dans le cadre d'un lotissement (approuvé) et finalement réalisée dans le cadre d'une ZAC.

\section{Une production de données géographiques homogénéisées dans le temps et l'espace}

11 Une des vocations de l'ensemble de ces données est d'être consultées par les chargés d'études de l'Agence d'urbanisme pour le développement de l'agglomération lyonnaise, dans le cadre de leur mission de diagnostic du territoire (projets de territoires, plans d'aménagement et de développement durable, etc.). Or, ceux-ci n'ont aucune compétence approfondie dans l'utilisation et la gestion de données dans un SIG. Il est important de pouvoir s'affranchir de l'interface du SIG et de permettre à chacun d'avoir accès à des représentations cartographiques homogènes, à des données statistiques précisant les évolutions observées avec la cartographie et, enfin, à des informations détaillées sur les processus ayant conduit à la structuration urbaine contemporaine.

Figure 1. Nomenclature utilisée pour l'étude de l'urbanisation de l'agglomération lyonnaise de 1950 à 2010

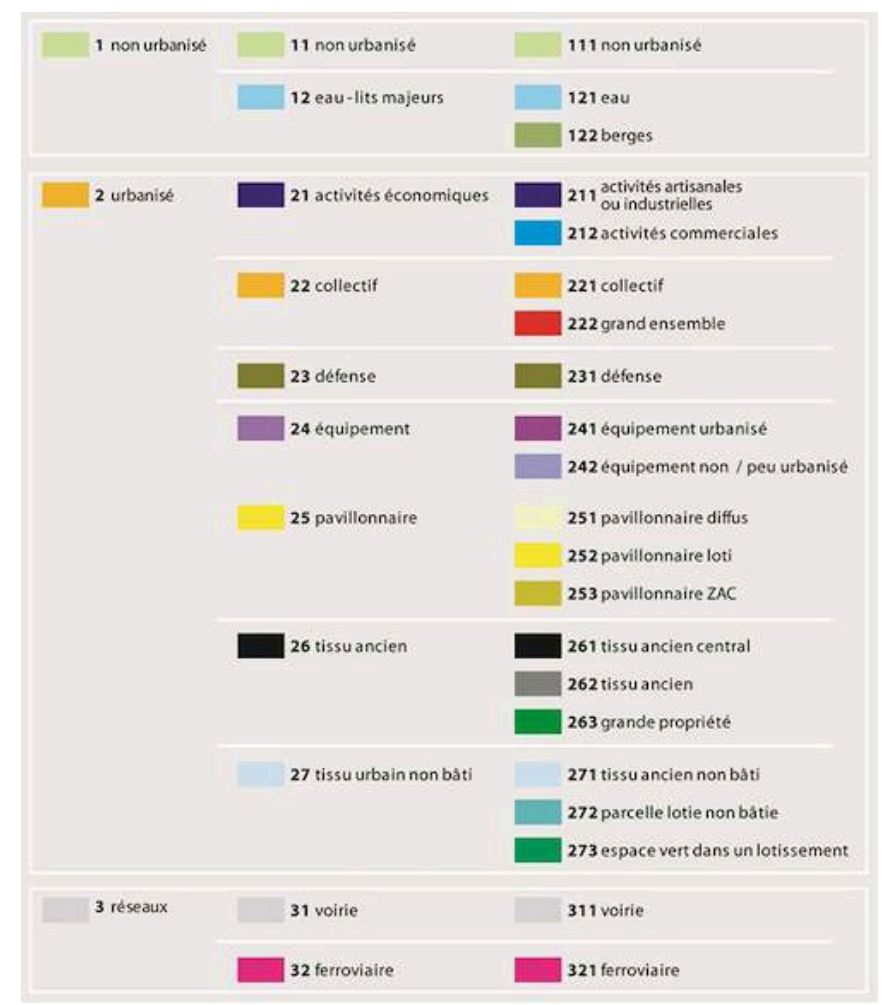

12 Un outil spécifique a été conçu en prenant exemple sur des modèles existants tels que, notamment, Corine Land Cover et Spot Thema Une nomenclature unique décrit de manière homogène, à l'échelle de la parcelle ${ }^{5}$, le territoire de l'agglomération, à partir 
de l'ensemble des bases de données existantes. Cette nomenclature se décline en trois niveaux :

- niveau 1 : il distingue uniquement les zones urbanisées, celles qui ne le sont pas et les réseaux, afin de visualiser la tache urbaine de l'agglomération ;

- niveau 2 : plus précis, il permet de distinguer, pour les zones urbanisées, le tissu ancien (qui n'a pas muté depuis 1950), les unités de logements pavillonnaires et collectifs, les équipements, les activités économiques et les emprises militaires ;

- niveau 3 : il est constitué de 21 postes de légende et offre la précision maximale disponible dans cette nomenclature. L'habitat pavillonnaire se décline, par exemple, en trois postes distincts, selon qu'il est réalisé dans un lotissement, une ZAC ou en diffus.

Figure 2. Expansion urbaine de l'agglomération lyonnaise de 1950 à 2010
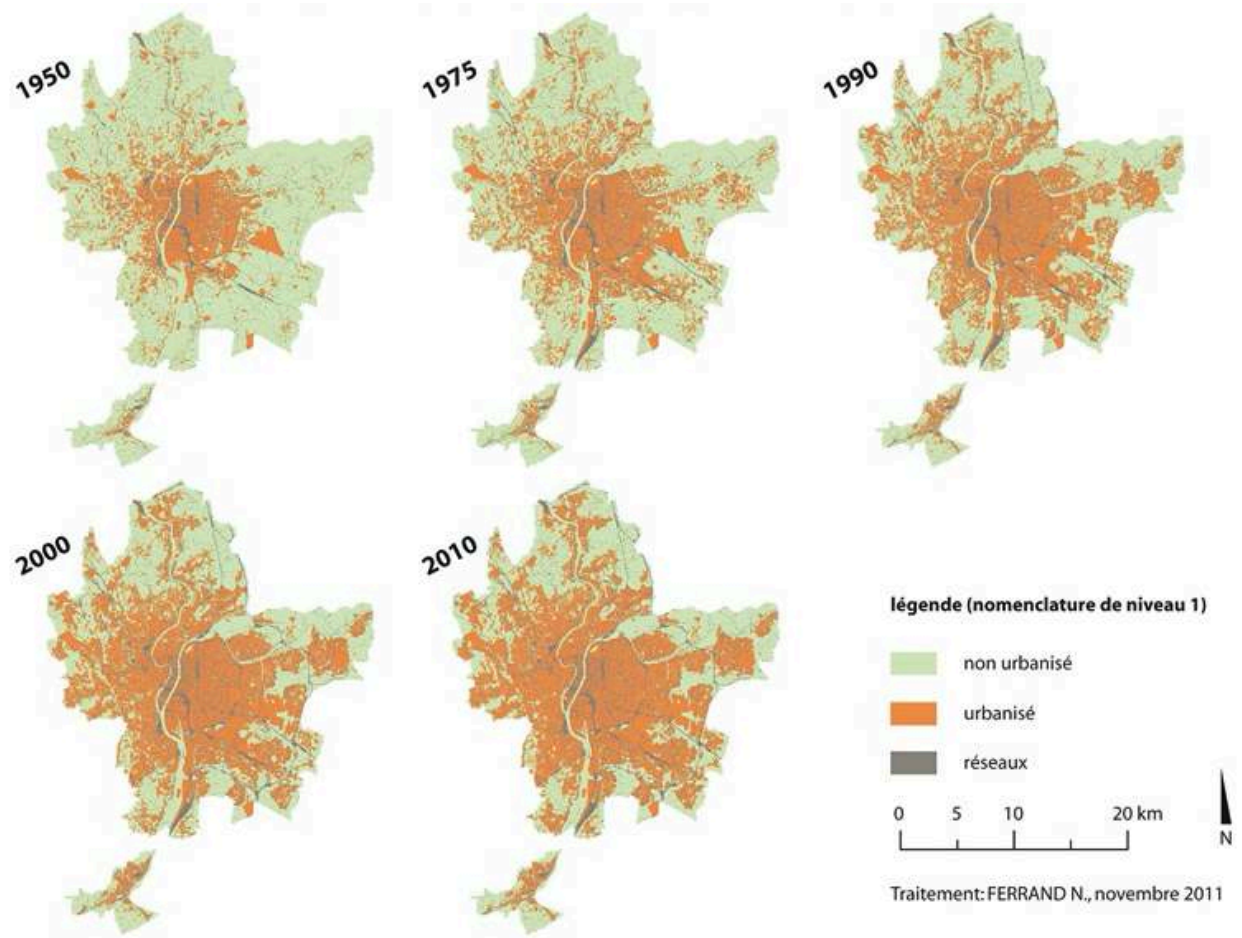

La dimension historique est systématiquement prise en compte, puisque ces trois rangs de nomenclature sont déclinés pour cinq périodes spécifiques : 1950, 1975, 1990, 2000 et 2010. La définition de ces cinq époques coïncide avec la disponibilité des couvertures cartographiques IGN, sur l'ensemble de l'agglomération. Des collections de cartes, centrées sur tout ou partie du territoire de l'agglomération, quel que soit le niveau de la nomenclature, permettent de visualiser les cinq états successifs de l'urbanisation. Ces collections de cartes peuvent également être mises en valeur sous forme de cartographie dynamique ${ }^{6}$.

Cet outil, calibré pour produire des cartographies homogènes dans le temps et dans l'espace, permet également le suivi des consommations de surfaces, par période et quel que soit le niveau de nomenclature. L'évolution de la tache urbaine, outre sa représentation graphique, peut ainsi être évaluée en fonction du foncier consommé depuis 1950, à l'hectare. Des tableaux croisés permettent de calculer, pour chaque pas de temps (1950-1975, 1975-1990, etc.), les surfaces urbanisées ou rénovées. Les différents types de surfaces sont ceux définis dans la nomenclature utilisée pour les 
représentations graphiques. Toutes les possibilités de mutation d'un type d'espace vers un autre, au cours de la période, sont ainsi calculées.

La base de données utilisée pour les exploitations cartographiques et statistiques contient également un certain nombre d'informations complémentaires, issues des bases de données constituées préalablement. Sont ainsi identifiées, par exemple, les procédures administratives recensées dans les bases sur les lotissements et les ZAC. Il est possible, pour une parcelle concernée par un lotissement de consulter, dans la base de données source, la totalité de l'information saisie lors du dépouillement ${ }^{7}$. Si les informations obtenues ne sont pas suffisantes, il restera la solution de consulter le dossier papier dans le fonds d'archives dans lequel il est conservé, à partir des références consignées dans cette base de données.

Figure 3. Évolution, entre 1950 et 2010 , de la structuration de l'agglomération lyonnaise (périmètre de la communauté urbaine de Lyon) en fonction de la nomenclature de niveau 2

\begin{tabular}{|c|c|c|c|c|c|c|c|c|c|c|c|c|c|}
\hline \multirow{2}{*}{\multicolumn{2}{|c|}{ HECTARES }} & \multicolumn{12}{|c|}{2010} \\
\hline & & activites & collectif & defense & $\begin{array}{l}\text { eaunflits } \\
\text { majeurs }\end{array}$ & equipement & ferroviaire & non urbanise & pavillonnaire & tissu ancien & $\begin{array}{c}\text { tissu urbain } \\
\text { non bâti }\end{array}$ & voirie & Total gentral \\
\hline & activites & 1158 & 229 & & 3 & 72 & & 9 & 7 & & 6 & 29 & 1513 \\
\hline & collectif & & 94 & & & & & & 1 & & & & 95 \\
\hline & defense & 76 & 10 & 291 & & 148 & & & & 2 & & 30 & 558 \\
\hline & eau-lits majeurs & 10 & & & 1660 & 10 & & 21 & & & & 44 & 1747 \\
\hline & equipement & 168 & 190 & & 3 & 1329 & 1 & 93 & 113 & & 38 & 70 & 2005 \\
\hline $\begin{array}{l}1 \\
9\end{array}$ & ferroviaire & 16 & 12 & & & 1 & 710 & $"$ & 1 & & 2 & 16 & 769 \\
\hline 0 & non urbanisé & 3514 & 1596 & 42 & 506 & 1610 & 174 & 18375 & 6631 & 6 & 697 & 2106 & 35257 \\
\hline & pavillonnaire & 1 & 8 & & & 2 & & & 1081 & 1 & & 4 & 1097 \\
\hline & tissu ancien & 176 & 873 & & 2 & 426 & & 31 & 286 & 3141 & 24 & 60 & 5019 \\
\hline & $\begin{array}{c}\text { tissu urbain non } \\
\text { bati }\end{array}$ & 91 & 64 & & 4 & 56 & 1 & & 31 & & 190 & 47 & 485 \\
\hline & volitie & 15 & 14 & 1 & 8 & 11 & 2 & 16 & 3 & 1 & 4 & 3314 & 3390 \\
\hline & Total général & 5225 & 3090 & 334 & 2187 & 3667 & 889 & 18557 & 8153 & 3151 & 961 & 5720 & 51935 \\
\hline
\end{tabular}

\section{Réinterpréter l'urbanisation de l'agglomération lyonnaise}

16 L'ensemble des données compilées et homogénéisées dans l'outil développé pour l'Agence d'urbanisme pour le développement de l'agglomération lyonnaise permet de chiffrer très précisément les mutations du paysage urbain depuis 1950. Celles-ci peuvent être classées dans deux grandes catégories : l'extension de l'urbanisation sur du foncier qui n'était pas construit antérieurement et le renouvellement de la ville sur elle-même.

\section{L'étalement pavillonnaire dans l'agglomération lyonnaise depuis} 1950

17 L'étalement de l'urbanisation de l'agglomération lyonnaise a été considérable depuis 1950. Son empreinte spatiale est passée de 11000 hectares en 1950 à plus de 24000 ha $^{8}$ en 2010. Alors que ces zones urbanisées ont plus que doublé, la population de l'agglomération est passée de 750000 à 1250000 habitants sur la même période, soit une augmentation de $66 \%$ (Insee, 2008). L'étalement urbain constitue donc bien un fait marquant de ces soixante dernières années. Deux principaux types d'unités urbaines méritent d'être remarqués, car ils induisent la création de paysages particuliers. Il 
s'agit tout d'abord du pavillonnaire, qui occupe huit fois plus d'espace en 2010 qu'en 1950. La deuxième plus grosse consommation foncière est constituée par les zones d'activités industrielles, artisanales et commerciales, dont l'empreinte spatiale a été multipliée par 2,5 depuis 1950.

Le phénomène pavillonnaire peut être considéré (du point de vue de l'utilisation de l'espace) comme un important gaspillage foncier dans l'agglomération lyonnaise. En 2010, 8150 hectares, soit 15,7\% de la surface de la communauté urbaine de Lyon, sont occupés par de l'habitat pavillonnaire (réalisé en diffus, dans le cadre de ZAC ou de lotissement). Si l'on ne considère que les zones urbanisées (33000 hectares sur les 52000 de la communauté urbaine), cette proportion est de $24,4 \%$. Or, sur l'ensemble de ces zones pavillonnaires, on dénombre environ 60000 logements, soit $10 \%$ du nombre total de logements recensés sur le territoire de l'agglomération (Insee, 2008). Certaines communes de l'agglomération sont particulièrement concernées par cette dynamique. À Jonage, Mions, Saint-Genis-les-Ollières, Solaize ou la Tour-de-Salvagny (des communes toutes situées à environ 10 kilomètres du centre de l'agglomération et formant sa dernière couronne de banlieue), plus des deux tiers des logements construits depuis 1950 sont des logements pavillonnaires.

19 Pour autant, la dynamique pavillonnaire est loin d'être uniforme sur toute l'agglomération lyonnaise. À l'est de Lyon, on constate une explosion du phénomène à partir des années 1970, sur d'anciennes zones agricoles ouvertes. Les unités pavillonnaires ont été constituées en grande majorité dans le cadre d'opérations de grande ampleur. Les paysages constitués dans ce contexte sont monotones, avec un habitat uniformisé dans de vastes périmètres. Cette impression est confirmée par le fait qu'un nombre limité de sociétés de promotion foncière et de cabinets de géomètres différents se sont partagé les réalisations dans ces secteurs. Les divisions parcellaires et les conceptions de programmes pavillonnaires ont ainsi été réalisées souvent de la même manière. Un autre trait de ce développement pavillonnaire, dans l'est de l'agglomération, est la relative petite taille des parcelles créées lors des divisions foncières, par rapport au nord et à l'ouest de celle-ci, notamment.

Dans le reste de l'agglomération, les manifestations de l'expansion pavillonnaire sont très différentes. Les implantations diffuses sont beaucoup plus nombreuses. Les opérations pavillonnaires sont généralement d'importance plus modeste, alors que la taille moyenne des lots est plus importante. La taille des parcelles peut être corrélée avec les revenus moyens des ménages. Les communes dont les ménages ont les revenus les plus élevés sont celles dans lesquelles les parcelles pavillonnaires sont les plus grandes. À titre d'exemple, à Vaulx-en-Velin (banlieue est de première couronne), la taille moyenne d'une parcelle pavillonnaire est de $600 \mathrm{~m}^{2}$, alors que le revenu net moyen par ménage est inférieur à $17500 €$ (Insee, 1999). Dans l'ouest de l'agglomération, à Charbonnières-les-Bains, les parcelles pavillonnaires ont une surface de $1720 \mathrm{~m}^{2}$ en moyenne, quand le revenu moyen des ménages de cette commune est supérieur à $20500 €$ (ibid.).

21 Les communes situées sur la rive droite du Rhône et de la Saône (Monts-d'Or, val d'Yzeron, etc.) accusent également un relief beaucoup plus contraignant que dans l'est lyonnais. Le paysage de ces communes n'apparaît pas comme aussi artificialisé que dans l'est, mais le mitage y est beaucoup plus important. Dans les communes des Monts-d'Or, au moins $50 \%$ de la surface urbanisée est concernée par de l'habitat pavillonnaire, diffus ou loti. En outre, l'initiative du lotissement n'est pas la même que 
dans les communes intensivement loties, dans l'est de l'agglomération. Il s'agit moins de programmes initiés par des sociétés de promotion foncière que d'initiatives privées, sur un foncier beaucoup plus fractionné à l'origine. De plus, un grand nombre de lotissements, dans cette partie de l'agglomération, a été réalisé dans le cadre de partages successoraux.

\section{Les activités industrielles et commerciales : deuxièmes plus grosses consommatrices d'espace depuis 1950}

La consommation d'espace par les activités, industrielles principalement, a également explosé dans l'agglomération lyonnaise depuis 1950. Au lendemain du second conflit mondial, les implantations industrielles sont héritées de logiques spatiales mises en place à la fin du XIX siècle (Laferrère, 1960). Les implantations industrielles, jusqu'au début des années 1970, se font de manière privilégiée le long ou à proximité des axes ferroviaires. Les implantations sont souvent réalisées le long de la ligne de chemin de fer Lyon-Grenoble, jusqu'à Saint-Priest (usines Berliet ${ }^{9}$ ), le long du chemin de fer de l'Est Lyonnais, plus au nord, sur les communes de Villeurbanne, Vaulx-en-Velin et Décines-Charpieu (société des Textiles artificiels du Sud-Est, Société lyonnaise de soies artificielles). À Lyon, les quartiers de Gerland et de Vaise, desservis par des embranchements ferroviaires et à proximité du Rhône et de la Saône, sont également des lieux de concentration des activités industrielles. Dans le sud de l'agglomération, à Givors et à Grigny, des sites industriels sont également implantés à proximité des voies ferrées (en direction de Marseille et de Saint-Étienne) et navigables (le Rhône et le Gier, dont l'emprise du canal sera réutilisée par l'autoroute A47 Lyon-Saint-Étienne, construite à partir de 1962).

À partir des années 1970, les logiques d'implantation des sites industriels de l'agglomération ne sont plus les mêmes. Comme cela a été prévu dans les documents de planification urbaine (plan d'urbanisme directeur de 1962, schéma Oream ${ }^{10}$ de 1967, etc.), l'industrialisation de l'agglomération se fait sur des sites qui ne sont plus choisis par l'industriel, en fonction d'opportunités foncières, mais par le planificateur, sur des sites desservis, désormais, par des infrastructures routières. Les surfaces dévolues aux activités ont plus que triplé, depuis 1950, passant, pour l'ensemble de l'agglomération, de 1500 à plus de 5200 hectares en 2010. Les plus importantes implantations ont été réalisées à l'est, sur les communes de Chassieu, Meyzieu, Mions, Corbas et Saint-Priest. Les zones d'aménagement concerté, créées dans le cadre de la loi d'orientation foncière de 1967, constituent le plus souvent la procédure mise en œuvre pour créer ces zones d'activités. On peut ainsi citer les ZAC de Chassieu Nord, approuvée par le conseil communautaire en 1975, des Gaulnes, à Meyzieu, approuvée en 1999, des Pierres Blanches à Mions, approuvée en 1994 ou celle du pôle alimentaire de Corbas, approuvée en 1998. Les périmètres de ces opérations sont tous compris entre 50 et 100 hectares. Ces zones industrielles se constituent sur un temps long. À leur création, peu d'entreprises y sont installées. Le SIG historique permet de visualiser la densification progressive de ces zones d'activités, qui s'achève, dans certains cas, plus de trente ans après leur création.

Le développement de zones commerciales aux entrées de l'agglomération est, enfin, un trait marquant de l'urbanisation lyonnaise. Un grand nombre d'implantations commerciales diffuses se sont implantées, depuis les années 1970, sur les axes 
structurants irriguant l'agglomération: le long des nationales 6 et 7, tant au nord (Dardilly, Champagne et Limonest) qu'à l'est (Bron, Saint-Priest, etc.). Des zones plus structurées ont été réalisées, dans la même logique que celles des implantations industrielles, dans le cadre de ZAC. C'est le cas de la ZAC de Dardilly, située le long de l'autoroute A6 et approuvée en 1983, dont l'objet principal était la construction d'un hypermarché. Il en est de même à Bron et à Saint-Priest, de la ZAC du Champ du Pont, approuvée en 1979. Cette opération, à proximité de grands ensembles (Bron-Parilly et Bron-Terraillon, notamment), est desservie directement par l'autoroute A43 (LyonGrenoble). Cette opération de plus de 40 hectares a permis l'implantation de grandes enseignes avec, pour certaines, un rayonnement régional (Ikea notamment). Un dernier exemple significatif est celui de la zone commerciale de Givors, réalisée en fond de vallée, entre le Gier et l'Autoroute A47, sur plus de deux kilomètres de long et environ cinquante hectares.

\section{Le renouvellement de la ville sur elle-même}

Alors que l'extension de l'agglomération peut être assez facilement observée et documentée, il n'en est pas forcément de même pour le renouvellement de l'agglomération sur elle-même, sur plus d'un demi-siècle. Des projets de rénovation urbaine ont été réalisés dès les années 1950. En 1960 a été présenté le plan " Maillet ${ }^{11}$ ", qui pointait notamment un grand nombre d'îlots du $3^{\mathrm{e}}$ et $\mathrm{du} 6^{\mathrm{e}}$ arrondissement, considérés insalubres, dont la rénovation était jugée nécessaire. La reconstruction de ces îlots se fera effectivement dans les années 1970 et 1980, notamment dans le cadre de la ZAC multisites Paul Bert, approuvée par le Grand Lyon en 1981. Cette ZAC a permis la réalisation d'un nouveau palais de justice de Lyon, à l'emplacement d'un îlot entier. Un autre exemple de rénovation emblématique est celle du quartier du Tonkin, à Villeurbanne, un quartier originellement organisé selon une trame orthogonale prolongeant celle du quartier des Brotteaux. Près de 40 hectares ont été entièrement rénovés, à partir des années 1970 . Dans ce cas également, la procédure utilisée par la puissance publique a été la ZAC. Quatre opérations successives (Tonkin tranche 1 à 3 et Charpennes-Wilson) ont été lancées entre 1972 et 1985. Le Grand Lyon, autorité compétente pour l'urbanisme, a délégué la réalisation de ces opérations à une société d'économie mixte, la Société d'équipement de la région lyonnaise.

La grande majorité du renouvellement urbain, au sein de l'agglomération, s'est réalisée, et se réalise toujours, à l'échelle de la parcelle ou du tènement foncier, hors de toute opération d'envergure. Le phénomène le plus marquant est la désindustrialisation, particulièrement pour Villeurbanne et pour les $3^{\mathrm{e}}, 7^{\mathrm{e}}$ et $8^{\mathrm{e}}$ arrondissements de Lyon. Le départ de certains établissements qui occupaient de grandes surfaces a parfois donné lieu à la réalisation d'une procédure de lotissement. Dans la majorité des cas, cependant, ces opérations, réalisées par des sociétés de promotion foncière, ne peuvent être documentées qu'avec le permis de construire. La connaissance de ces nombreuses mutations n'a pu être appréhendée qu'avec l'analyse méthodique des versions successives du plan général de la ville de Lyon au $5000^{\circ}$ (archives municipales de Lyon, série 5s) et des couvertures photographiques réalisées par l'Institut géographique national.

Dans le centre de l'agglomération, c'est-à-dire dans les communes de Lyon et de Villeurbanne, un quart de la surface a muté depuis 1950, soit 1450 hectares sur les 
6200 de ces deux communes. Or, sur ces 1450 hectares, seuls 380 n'étaient pas urbanisés en 1950. Plus de 1000 hectares correspondent à un renouvellement urbain qu'il est très difficile d'appréhender. L'utilisation des bases de données consolidées avec l'Agence d'urbanisme de Lyon permet d'avoir certains éléments sur le contenu de ce renouvellement urbain. On sait désormais que sur ces deux communes, 180 hectares occupés par des activités industrielles ou artisanales ont disparu pour être remplacés, dans plus des deux tiers des cas, par des unités de logements collectifs. De même, près de 150 hectares d'emprises militaires ont été déclassés depuis 1950, au profit d'un grand nombre d'équipements, au premier rang desquels le campus scientifique de la Doua, à la place d'un camp militaire, sur près de 70 hectares. Une autre réutilisation emblématique d'emprise militaire est celle de la caserne de la Part-Dieu, sur laquelle ont été construits le quartier d'affaires, l'hôtel de la communauté urbaine de Lyon, le centre commercial, ainsi que la bibliothèque de Lyon et l'auditorium Maurice-Ravel. Ce projet d'envergure a entraîné la rénovation de près de 30 hectares.

Figure 4. Parcelles qui, dans le centre de l'agglomération lyonnaise, ont muté en extension ou en renouvellement entre 1950 et 2010

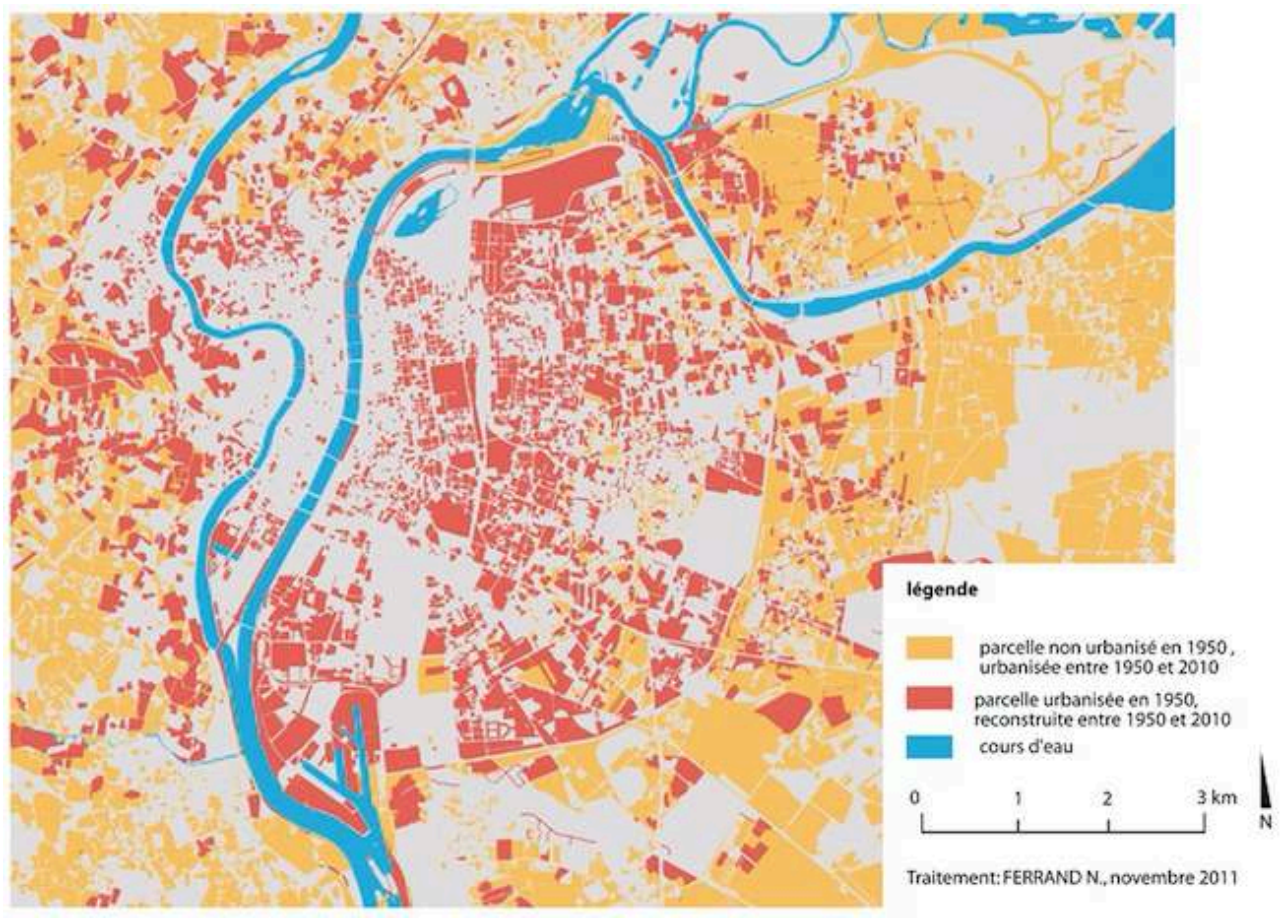

Les plus importantes transformations concernent cependant la destruction du tissu ancien, c'est-à-dire l'habitat résidentiel hérité, très majoritairement, de la fin du XIX $\mathrm{X}^{\mathrm{e}}$ et du début $\mathrm{du} \mathrm{xx}^{\mathrm{e}}$ siècle. 480 hectares ont ainsi changé de destination en soixante ans. Plus des deux tiers des emprises ont été mobilisées pour la construction de logements collectifs. 110 hectares ( $23 \%$ ) ont permis la construction d'équipements parmi lesquels, par exemple, un grand nombre d'établissements scolaires. Un dernier exemple significatif de la mutation du territoire du centre de l'agglomération concerne la division par cinq (de 250 à 50 hectares), sur l'ensemble de la période, des surfaces occupées par les grandes propriétés (maisons bourgeoises et châteaux établis sur un parc), très majoritairement au profit de programmes de construction de logements collectifs privés. La cartographie dynamique permet de suivre la réutilisation 
progressive des parcs pour l'implantation d'immeubles de logements, parfois sur des temporalités très longues.

La création d'un outil capable de suivre l'urbanisation de l'agglomération lyonnaise trouvait sa justification initiale dans un programme de recherche financé par la Région Rhône-Alpes, le Cluster 13 Patrimoine, Culture et Création ${ }^{12}$. Certains développements, à la fois des méthodes et de la production d'information géographique, ont été réalisés dans un cadre différent : celui de l'Agence d'urbanisme de Lyon, acteur majeur de la réflexion sur l'aménagement du territoire de Lyon et de son agglomération. Un avantage certain découle de l'appropriation de cet outil par une structure pérenne, productrice par ailleurs de certaines sources mobilisées en amont. Il s'agit de la possibilité de mettre à jour, périodiquement, l'ensemble des données compilées jusqu'à présent. L'objectif devenant non seulement la connaissance de la production urbaine passée mais également une veille quotidienne sur la transformation continue d'une agglomération de plus d'un million d'habitants.

En outre, il devient intéressant de relire les politiques d'aménagement conduites sur l'agglomération lyonnaise et d'en mesurer l'efficacité. Il est possible, par exemple, de vérifier si des objectifs de production de logements ou de préservation d'espaces naturels ont bien été réalisés, compte tenu des prescriptions formulées dans le plan d'urbanisme directeur de 1969, le schéma directeur de l'agglomération lyonnaise de 1992, etc. De nouvelles perspectives s'ouvrent enfin avec le plan local d'urbanisme, dont la révision générale vient d'être lancée récemment par le Grand Lyon. Son projet d'aménagement et de développement durable s'appuiera notamment sur des éléments de diagnostic issus de ce nouvel outil. Le nouveau PLU pourra enfin régulièrement être expertisé pour vérifier si l'urbanisation lyonnaise se concrétise selon les principes et prescriptions édictés par ce document.

\section{BIBLIOGRAPHIE}

Arnaud, J.-L., Analyse spatiale, cartographie et histoire urbaine, Paris, Parenthèses/MMSH, 2008, $233 \mathrm{p}$.

Beaujeu-Garnier, J., " Méthode d'étude pour le centre des villes ", Annales de géographie, $\mathrm{n}^{\circ}$ 406, vol. 74, 1965, p. 695-707.

Belliot, M., « Vive le lotissement ! ", Études foncières, n 100, novembre-décembre 2002, p. 25-26.

Bloch, M., «Les plans parcellaires en France », Annales d'histoire économique et sociale, $\mathrm{n}^{\circ}$ 1, 1929, p. 60-70.

Bonneville, M., Désindustrialisation et rénovation immobilière dans l'agglomération lyonnaise : le cas de Villeurbanne, Paris, l'Hermès, 1975, 136 p.

Bonneville, M., Villeurbanne : naissance et métamorphose d'une banlieue ouvrière, Lyon, Presses universitaires de Lyon, 1978, 296 p. 
Bonneville, M., « Planification et développement urbain dans l'agglomération lyonnaise : essai d'évaluation pour la période 1968-1982 ", Revue de géographie de Lyon, nº 2, vol. 57, 1982, p. 93-116. Bruyère, G., Chiron, N., Dureau, J.-M., Forma Urbis : les plans généraux de Lyon, $\mathrm{XVI}^{e}-\mathrm{XX}{ }^{e}$ siècle, Lyon, Archives municipales, 1997, $249 \mathrm{p}$.

Chaline, C., La Régénération urbaine, Paris, PUF, 1999, 127 p.

Delfante, C., 100 ans d'urbanisme à Lyon, Lyon, Lugd, 1994, 233 p.

Demangeon, A., « Les recherches géographiques dans les archives », Annales de géographie, $\mathrm{n}^{\circ}$ 87, 1907, p. 193-203.

Duby, G., (sous la dir de), Histoire de la France urbaine. La ville aujourd'hui, t. V, Paris, Seuil, 1985, $668 \mathrm{p}$.

Fédération nationale des agences d'urbanisme, Habitat et formes urbaines. Densités comparées et tendances d'évolution en France, Paris, FNAU, 2006, 276 p.

Gauthiez, B., Espace urbain : vocabulaire et morphologie, Paris, Monum, Éditions du patrimoine, 2003, $493 \mathrm{p}$.

Gauthiez, B., Zeller, O., « Espace construit, espace social à Lyon aux XVII ${ }^{\mathrm{e}}$-XIX ${ }^{\mathrm{e}}$ siècle : l'apport du SIG », dans Panzeri, M., Ferrugia, I. (dir.), Fonti, Metafonti e GIS per l'indagine della strutura storica del territorio, Turin, Celid, 2009, p. 39-49.

Gages, R., Grimal, G., Tourret, P., « Reconstruction à Lyon », L'Architecture d'aujourd'hui, nº 32, novembre 1950, p. 33-37.

George, P., « Géographie et urbanisme », Annales de géographie, nº 406, vol. 74, 1965, p. 641-659.

Gregory, I., Healey, R., « Historical GIS : structuring, mapping and analyzing geographies of the past », Progress in human Geography, n 31, 2007, p. 638-653.

Gregory, I., Kemp, K., Mostern, R., « Geographic Information and historical research : Current progress and future directions ", History and Computing, $\mathrm{n}^{\circ} 13,2001$, p. 7-21.

Kleinclausz, A., Lyon des origines à nos jours, Lyon, Masson, 1925, $429 \mathrm{p}$.

Laferrère, M., Lyon ville industrielle, Paris, PUF, 1960, 548 p.

Panerai, Ph., Depaule, J.-C., Casteix, J., Formes urbaines, de l'îlot à la barre, Marseille, Parenthèses, 1997, 196 p.

Pelletier, J., Delfante, C., Atlas historique du Grand Lyon. Formes urbaines et paysages au fil du temps, Seyssinet-Pariset, Xavier Lejeune-Libris, 2004, 221 p.

Rouleau, B., Villages et faubourgs de l'ancien Paris, Paris, Seuil, 1985, 379 p.

\section{NOTES}

1. Ancêtre des Plans d'occupation des sols et des plans locaux d'urbanisme

2. Système d'information géographique.

3. Obligation imposée par la loi SRU, en 2000, pour recenser l'ensemble du parc locatif social.

4. L'agglomération est couverte de manière homogène au $1 / 25000^{\mathrm{e}}$ en 1950, 1975, 1990, 2000 et 2010.

5. Le Grand Lyon en compte près de 270000. 
6. Réalisées en Flash, GIF animé ou même à partir d'un simple logiciel de présentation type Microsoft Power Point.

7. En l'occurrence, pour un lotissement : l'adresse de l'opération, l'identification du lotisseur, du géomètre, la nature exacte du programme, etc.

8. Ces chiffres, comme tous ceux présentés ci-après, sont inédits. Ils sont issus de l'exploitation des bases de données consolidées en octobre 2011.

9. Qui deviendront Renault Véhicules industriels puis Renault Trucks.

10. Organisation des études d'aménagement de l'aire métropolitaine.

11. Du nom de son concepteur, Joseph Maillet, architecte-urbaniste chargé d'élaborer le projet d'urbanisme communal de Lyon, à partir de 1956.

12. http://cluster13.ens-lyon.fr/.

\section{RÉSUMÉS}

L'étude de l'urbanisation de l'agglomération lyonnaise à l'échelle parcellaire repose sur l'identification et la mobilisation d'un grand nombre d'archives et de sources hétérogènes. Un des enjeux pour la constitution d'un nouvel outil d'analyse de l'urbanisation repose sur l'intégration de ces données dans un ensemble cohérent, grâce à un Système d'information géographique (SIG). Ce nouvel outil permet de suivre, à l'hectare, l'évolution de l'urbanisation, en fonction de ses caractéristiques morphologiques depuis 1950. Il s'agit de retracer l'expansion urbaine, mais aussi le renouvellement de la ville sur elle-même. Les derniers développements de cet outil ont été réalisés en collaboration avec l'Agence d'urbanisme pour le développement de l'agglomération lyonnaise.

The study of the urbanization of the Lyon area is based on identifying and mobilization of large numbers of archives and heterogeneous sources. One of the challenges for the establishment of a new tool for analysis the urban sprawl is based on integration of these data into a coherent whole, with a Geographical Information System (GIS). This new tool allows tracking per hectare, the development of urbanization, based on its morphological characteristics since 1950. The aim is to track urban sprawl but also the renewal of the city to itself. The latest developments of this tool have been made in collaboration with the Lyon area urbanism agency.

\section{INDEX}

Mots-clés : archives, étalement urbain, Système d'information géographique, SIG, urbanisation, ville

Keywords : archives, urban sprawl, Geographical Information System, GIS, urbanization, city

\section{AUTEUR}

\section{NICOLAS FERRAND}

Chargé d'études à l'Agence d'urbanisme pour le développement de l'agglomération lyonnaise, Nicolas Ferrand est licencié en histoire et en géographie, et docteur en géographie et 
aménagement (thèse soutenue en juin 2010 à l'université Jean Moulin Lyon 3, CNRS UMR 5600 Environnement, Ville et Société).

ferrandnicolas[at]hotmail[dot]fr 